\title{
The Mechanisms Morpho-Lexical and Semantic, that Bring such Language Solutions. The Formation of Words with Prefixes and Suffixes, Phenomena and its Peculiar Aspects in Italian
}

\author{
Arben Skendaj Ph.D. \\ Department of Italian, Faculty of Foreign Languages, University of Tirana \\ Email: arskendaj@hotmail.com
}

Doi:10.5901/mjss.2013.v4n3p361

\section{Abstract}

Word-formation in Italian as a lexical and linguistic phenomenon is essential to the enrichment of Italian language and not only. Its various aspects consider the prefixes, the suffixes and the composition. On one hand, there is the derivating ratio of the composition and on the other hand, we deal with the lexicology and semantics. Also, I am taking into consideration the report of prefixes and suffixes in Italian, focusing on the synchronic and diachronic studies in general, and particularly on the vocabulary. The subject of my analyses is the interfering of linguistic phenomena, focusing on the cases of derivation, but at the same time intertwining these two types and case studies, giving the value of prefixes and suffixes in word-formation. Prefixes, suffixes and composition are not only some ways to resolve powerful linguistic problems, but also serve to enrich the vocabulary of Italian, and any other language.

Keywords, prefix, suffix, derivation, word, meaning.

La lingua italiana in fatto di formazione di parole e di derivazione è una delle lingue, che ha un sistema così complesso, completo ed elastico, che forse poche lingue al mondo avrebbero e che glielo invidierebbero ad averlo. Questo si può intuire, dal fatto, che in buona parte si deve anche al che, l'italiano eredita una buona parte del patrimonio linguistico del latino. E questa idea dell'ereditarietà, va riferita e collegata anche al fatto della formazione delle parole e dei mezzi di formazione quali prefissi e suffissi. Qui non va dimenticato anche l'altra parte del capitolo della formazione delle parole, che è il capitolo della composizione. Come sappiamo già, esso fa parte del maxisistema della formazione delle parole, però è relativamente abbastanza più semplice per ragioni considerate note, e in parte le chiariremo appena sotto.

Stabiliamo subito il posto, il valore e il suo rapporto in seno alla formazione delle parole. La composizione, comunque in linea generale va detto che è relativamente più semplice per alcuni motivi; uno, perché è relativamente meno produttivo della formazione delle parole con prefissi e suffissi, numericamente parlando. Unità lessicali formate con due unità autonome semanticamente, è un fenomeno poco produttivo in italiano, secondo, la sua problematicità semantica presenta poca complessità e forse la ragione di tutto questo è che la composizione produce parole un po "gravide" e scomode per la loro poca pratica struttura formale fonetica morfologica e lessicale, che sono appunto due 0 più parole diverse messe insieme dalla esigenza semantica di formare un concetto solo. La composizione pur manifestandosi relativamente semplice come un sottosistema di formazione delle parole a volte, visto le ragioni lessicosemantiche, per cui si formano le parole, risulterebbe essere indispensabile nella lingua come soluzione. Sembra che debba essere considerata come una via estrema per risolvere semanticamente problemi lessicali, cioè la creazione di nuove parole.

A volte la situazione però potrebbe sembrare strana, perché l'italiano in contesti simili qualche volta preferisce formare una nuova parola creando un neologismo con l'aiuto di un suffisso o prefisso e a volte non si sa su casi identici, perché preferisce la via della composizione invece che della formazione delle parole con suffissi e prefissi. Le ragioni si possono intuire che possano essere da quelle linguistiche a quelle sociali, antropologiche, soggettive, forse preferenze di persone o gruppi sociali, che fanno attecchire casualmente un neologismo formato in un modo invece in un altro. Per esempio non si può capire, perché quando è stato creato il neologismo schiaccianoci è stata preferita la via della composizione, mentre quando è stato creato il neologismo frullatore è stata preferita la via della formazione del neologismo col suffisso, se per esempio potevamo benissimo formare il composto frullafrutta. Oppure il contrario, dire lo schiacciatore per l'arnese che serve per schiacciare le noci e frullafrutta per l'arnese che serve per frullare le frutta. Casi così possiamo trovare a bizzeffe in italiano e potremmo discuterne a voglia, ma il problema consiste nell'analizzare, capire e conoscere i composti a livello lessicale. Comunque rinnoviamo l'idea, che i composti nella lingua italiana e in 
molte lingue sono un fenomeno indispensabile, seppur limitato e meno preferito rispetto alla prefissazione e suffissazione. Morfologicamente parlando troviamo una situazione abbastanza variegata, quanto alla formazione di parole, cioè troviamo un verbo più un sostantivo, che è anche uno dei gruppi più nutriti di esempi per esempio; temperalapis, aspirapolvere, parafango, appendiabiti, cavatappi, apriscatole, battimano, batticuore, lustrascarpe ecc. Vediamo altri casi dove si costata la presenza di due sostantivi per esempio testacoda, pescespada, pescecane, madreperla, terremoto, arcobaleno. Un altro caso è quello di unione di una preposizione e un sostantivo per esempio sottoaceto, oltretomba ecc. II caso di viavai è un caso formato da sostantivo che è via 0 anche avverbio di luogo più verbo all'imperativo vai di andare. II caso di mezzogiorno è un'unione tra aggettivo e sostantivo, invece cassaforte è formata da sostantivo cassa e l'aggettivo forte. La parola camposanto è formata da sostantivo campo più aggettivo santo. Cassaforte è stata formata da un sostantivo più un aggettivo cassa e forte, biancospino, aggettivo bianco più il sostantivo spino, agrodolce, aggettivo più aggettivo agro e dolce come pure rossonero, grigioverde ecc. Belvedere è un'unione di aggettivo più verbo bello e vedere. Dormiveglia, fuggifuggi, saliscendi sono due verbi uniti dormire e vegliare come pure fuggire, appare due volte nella formazione di un composto e anche salire più scendere. Posapiano si riconosce che è stata formata dal verbo posare più avverbio di modo piano. Benestare è l'unione di un avverbio di modo bene più il verbo stare. Girasole è formato dal verbo girare e più il sostantivo sole. Soprannome è formata da sopra più il sostantivo nome, terracotta da sostantivo terra più aggettivo cotta.

Dopo questa carrelata modesta di esempi possiamo pure suggerire, che teoricamente dal punto di vista semantico i composti dovrebbero essere il significato derivato matematicamente dall'aggiunta di due concetti necessari, ma a volte si intuisce, che il significato non si ottiene così meccanicamente, ma che a volte una delle due parole compromette il suo significato originale in funzione del significato, che si vuole avere col nuovo neologismo. Poi un'altra idea da accentuare è che nel caso di sottostare, oltretomba, sottaceto ecc. il primo elemento è un po' contraddittorio, per quanto riguarda il concetto di base della composizione, lo mette un po' in crisi. Questo perché, noi abbiamo sostenuto che la composizione è il caso della formazione delle parole derivata dall'unione di due unità semanticamente parlando autonome, ma il caso succitato pare, che il primo elemento non corrisponderebbe a una parola semanticamente autonoma. Sopra sotto e oltre non sarebbero parole vere e proprie; alcune grammatiche le citano come prefissi latini, per cui già qui siamo un po' imprecisi, perchè come tali esse non farebbero parte della grammatica attiva dell'italiano, ma apparterrebero a una visione diacronica della lingua, etimologica, per cui, meno coerente con la trattazione dell'italiano moderno. Qualche altra grammatica le cita come preposizioni, che mi sembra più idonea come definizione. Questa visione di analisi è sincronica, dinamica, per cui più contemporanea con lo studio dell'italiano. Dopo questa parentesi doverosa sulla composizione, un po' lunga, ma inevitabile fa fare torniamo adesso all'idea dell'inizio, del perché la lingua italiana presenterebbe un sistema così complesso e completo, per quanto riguarda la formazione delle parole, suggerito sopra. Credo che ci siano più fattori da me supposti; uno che l'italiano è la diretta discendenza del latino, tale che io nelle mie lezioni spesso l'ho considerata anche quasi figurativamente come il "latino moderno". Senza dubbio che l'italiano appartiene alla famiglie delle lingue neolatine insieme al francese, spagnolo, portoghese e rumeno. Però il fatto storico-geografico dell'Italia, che si trova nello stesso territorio naturale dove ha avuto la vita e lo svolgimento dell'Impero più grande di tutti i tempi, dell'Impero romano, che è stato usato appunto come lingua di comunicazione e di cultura il latino, forse per queste ragioni la lingua italiana presenterebbe oggi una più genuina, pura, naturale, quasi trascendentale ereditarietà dal latino rispetto alle altre lingue neolatine. Questo credo, che sia il primo fattore di questo rapporto privilegiato dell'italiano col latino a giustificare questa ricchezza del meccanismo di formazione delle parole che ha l'italiano. Un altro elemento sarebbe anche il fatto che l'italiano è stata la lingua di utilizzazione e di trasferimento di tutta la cultura del Risorgimento italiano, che ebbe inizio a Firenze, che come sappiamo influenzò lo sviluppo rinascimentale di tutta l'Europa di allora.

Questa parentesi un po' storica e culturale, pure doverosa per poter capire e spiegare fenomeni, che sono di natura tutt'altra che tale, cioè che sono di natura prettamente linguistica.

La formazione delle parole come meccanismo lessico-semantico è una via molto importante nel risolvere problemi lessico-linguistici e nell'arricchire il lessico dell'italiano. Come fenomeno la formazione delle parole è molto complesso ed ha molti casi diversi di sottomeccanismi grandi e piccoli a creare parole nuove. lo ho preferito in questa analisi, non a caso, a discutere ed analizzare il caso della formazione delle parole, che riguarda tra l'altro anche la suffissazione nominale. II caso di formazione di parole e di derivazione e qui il caso delle parole astratte è molto complicato e io ragiono, che essendo il caso più complicato di derivazione questo semplificherebbe a capire i restanti casi di derivazione di parole.

La derivazione è un concetto vasto e prima di tutto, è un fenomeno morfologico e poi in secondo luogo è sempre una ragione semantica, che la motiva. In effetti la semantica nei casi di derivazione si giustifica per prima, perché nella lingua e nella comunicazione è la logica, cioè la semantica, che giustifica la nascita di una parola, poi la realizzazione 
lessico-linguistica è una soluzione piuttosto pratica. La sua realizzazione pratica non è sempre facile, a volte è risultata molto difficile.

La formazione delle parole è un concetto vasto, che racchiude la derivazione, la composizione, e i casi nei quali un elemento formativo non esiste proprio, cioè è zero, e si avverte pure la creazione di una nuova parola, e quindi la creazione di un nuovo concetto. La derivazione sottintende la formazione delle parole tramite l'unione di un tema con un prefisso o con un suffisso o con prefissi e suffissi contemporaneamente.

La suffissazione è nominale, verbale, aggettivale, vediamo un po' la suffissazione nominale per le ragioni suddette, ed anche perché più analizzando casi specifici più è più facile capire ed analizzare un fenomeno intero. "Nello studio della formazione delle parole si deve tenere conto di due fattori: la motivazione del procedimento formativo e la sua trasparenza:" (Serianni Luca, Grammatica italiana, pagina 635 ).

La suffissazione nominale in italiano presenta molti casi di elementi formativi, cioè suffissi diversi. In questa moltitudine di suffissi bisogna distinguere i suffissi nominali importanti, frequenti e complicati semanticamente o meno. Questo aspetto di suffissazione nominale, cioè la semantica presenta nella formazione delle parole e nella derivazione coi suffissi il caso più intricato e questo è anche uno dei motivi, perché ho preferito occuparmi, tra l'altro anche di questo caso di derivazione ed analizzare queste unità lessicali.

Così per esempio da scuola col suffisso -aro abbiamo scolaro, da tenda col suffisso -poli abbiamo tendopoli, da polizia col suffisso -esco abbiamo poliziesco, da guerra abbiamo formato guerresco, guerrafondaio, possiamo citare liberamente premuroso, increscioso, fermentazione, discussione, ingrandimento, lavorazione, dibattimento, dichiarazione, diffidenza, bravura, chiusura, eleganza, digeribile ecc. Vediamo dei derivati coi prefissi, così da prendibile abbiamo derivato col prefisso negativo -in, imprendibile dove diventa $\mathrm{m}$ la $\mathrm{n}$ del prefisso ovviamente, perché sappiamo che viene assimilata foneticamente dal contatto con la lettera $p$, e questo non è assolutamente l'unico caso di assimilazione di lettere a contatto di lettere determinate in italiano, ma non ci allunghiamo più su questo. Poi con vari prefissi possiamo citare, solo a titolo di esempi, casi liberi come inenarrabile, indicibile, ineffabile, incredibile, indiscutibile, scarcerare, imprigionare, incutere, incriminare, incatenare, digradare, dispartire, dilungarsi, dipendere, diramare, discendere, derivare, detrarre, deformare, decomporre, degenerare, disperdere, disporre, distogliere, distrarre, distribuire, disfare, dispiacere, disaccordo, disadorno, discordanza ecc,

Quanto ai prefissi e i suffissi bisogna ricordare che questi elementi formativi, oltre ad avere un inevitabile rapporto con la semantica, hanno assolutamente un rapporto anche con la morfologia. II mio punto di vista è una visione di natura prevalentemente lessico-semantica, ma spesso per capire meglio un caso 0 un altro di preffissazione e di suffissazione, mi dovrò riferire anche alla morfologia.

Spesso costatiamo, che abbiamo una parola italiana derivata dal latino e vediamo che, possa avere una famiglia derivativa, la cui situazione etimologica va analizzata a seconda del caso specifico, questo, perché le parole derivate poi, vediamo, che alcune di queste parole sono derivate in diversi periodi di tempo, pur avendo una base iniziale di origine latina. A volte possiamo trovare una famiglia di parole derivata tutta intera dal latino, per cui bisogna sempre indagare da caso a caso. Vediamo per esempio solo a caso la famiglia della parola medicare. Uno dei maggiori vocabolari di autorità indiscussa lessicografia è ovviamente Treccani "L'oggetto della lessicografia è chiarire cosa una parola denoti" (Chierchia, G. (1997). La semantica, le strutture della lingua, pagina 195)

medicare v. tr. [dal lat. medicare, der. di medícus «medico2»] (io mèdico, tu mèdichi, ecc.). - 1. Eseguire su una parte del corpo il trattamento terapeutico locale adatto a proteggere tessuti lesionati e a favorirne la cicatrizzazione 0 in genere i processi riparativi (v. medicazione): $m$. una ferita, una contusione, un ascesso; farsi $m$. un dito, un dente, un orecchio; anche riferito alla persona: $m$. i feriti; $m$. un paziente operato. 2. estens. Trattare con aggiunta di sostanze antisettiche $0 . .$.

medicazióne s. f. [dal lat. medicatio -onis, der. di medicare «medicare»]. - 1. a. Terapia locale intesa a proteggere $i$ tessuti lesionati e a favorirne $i$ processi riparativi: fare, farsi una $m$.; $m$. antisettica, attuata con cotone o garze impregnati di antisettici; $m$. asettica, eseguita con materiale sterile, e attualmente preferita perché meno irritante per $i$ tessuti lesionati. Nel linguaggio milit., posto di m., formazione sanitaria militare di prima linea, che provvede alla medicazione delle...

medicaménto s. m. [dal lat. medicamentum, der. di medicare «medicare»]. - 1. Ogni sostanza o preparato usati a scopo terapeutico, in partic. quelli usati per favorire i processi riparativi di una lesione: la ferita era ormai infetta, e ogni $m$. si rivelò inutile. Non com. in senso fig., rimedio o sollievo a mali e sofferenze morali: non c'è m. efficace per le delusioni d'amore. 2. Raro in senso astratto, l'azione del medicare, medicazione.... 
medicatóre s. m. (f. -trice) [dal lat. tardo medicator -oris], raro. - Chi, o che, medica; soprattutto in senso fig.: il tempo è il vero $m$. delle piaghe dell'animo; anche con funzione di agg.: le forze $m$. della natura..

medicàbile agg. [dal lat. medicabilis]. - Che può essere medicato: ferita m.; in senso fig.: dolori facilmente m.; una ferita amorosa non medicabile...

mèdico3 s. m. [dal lat. medĭcus, propr. uso sostantivato dell'agg. prec.] (pl. -ci). - 1. Chi professa la medicina: albo, ordine dei m.; fare il m., esercitarne la professione; chiamare il m. (pop. andare per il m.); consultare il $\mathrm{m}$. o un $\mathrm{m}$.; l'opera, il consiglio, il parere del m.; un buon m., un cattivo $m$. (con riguardo alle attitudini, alla capacità); un m. bravo, valente, famoso; $m$. di casa, di famiglia (attualmente, $m$. di famiglia sostituisce la precedente denominazione di $m$. della mutua); $m$. curante, quello che ha in cura un paziente; $m$. consulente, quello chiamato per un consulto...

mèdico2 agg. [dal lat. medǐcus, der. del tema di mederi «curare»] (pl. m. -ci). - 1. a. Attinente alla medicina: scienza, arte $m$.; discipline $m$.; clinica $m$.; congresso m.; biblioteca m.; esperienza, pratica m.; cure mediche. Fisica m., disciplina che si occupa dell'applicazione dei principî e dei ritrovati della fisica nei varî settori della medicina, soprattutto nella diagnostica e nella terapia, con esclusione delle questioni riguardanti la protezione dell'organismo umano da radiazioni, delle quali si occupa la fisica sanitaria. Materia m., espressione usata un tempo come sinon...

medico-sentinella (medico sentinella), loc. s.le m. Medico incaricato dal ministero della Salute di sorvegliare con regolarità l'insorgenza e la progressione di malattie infettive e di segnalare eventuali effetti indesiderati prodotti da farmaci prescritti ai pazienti. [tit.] Virus, cordone sanitario in Italia / In autunno la polmonite atipica dovrebbe arrivare in Europa. Vertice per un piano di emergenza infettiva / Corsi di aggiornamento per medici-sentinella...

medico-clown (medico clown), loc. s.le m. Medico travestito da clown, che intrattiene i bambini ricoverati in strutture ospedaliere, per distrarli e suscitare in loro il buonumore. [tit.] Hanno ammazzato 30 ore per la vita / La maratona della [Lorella] Cuccarini sostituita da un'iniziativa ispirata al medico-clown Patch Adams (Libero, 2 agosto 2002, p. 21, Spettacoli) •

mèdica1 agg. e s. f. [dal lat. class. Medica (herba), gr. Mnঠıкń (пóa) «erba della Media»]. - Pianta perenne delle leguminose papiglionacee (Medicago sativa), detta più comunem. erba medica e anche erba Spagna, a rigoglioso sviluppo, con radice fittonante e profonda, fusti eretti, foglie composte trifogliate, fiori di colore variabile fra il violettoturchino e il celeste biancastro, e legumi avvolti a spirale; è una pianta largamente coltivata nei climi temperato-caldi, perché dà un foraggio di ottima qualità....

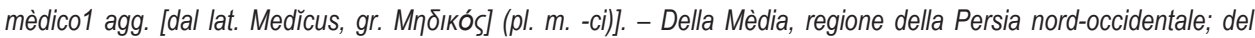
popolo dei Medi (v. medo): guerre m., quelle combattute dal 504 al 449 a. C. tra la Grecia e la Persia sotto Dario I e i suoi successori, più note come guerre persiane....

mèdica2 s. f. [dal lat. tardo medica; v. medico3], ant. - Donna che esercita la medicina: ella, che medica non era come che medico fosse il marito ... (Boccaccio); per estens., donna che cura un malato; anche, donna che pretende di avere capacità di guaritrice. Cfr. medichessa....

medice, cura te ipsum (mèdiče ...) (lat. «medico, cura te stesso»). - Proverbio che Gesù (Luca 4, 23) immagina possa essergli ricordato dagli abitanti di Nazareth quasi a esortarlo a compiere a Nazareth, sua patria, quelle azioni miracolose che si diceva avesse fatte a Cafarnao; ma Gesù replica all'obiezione con dolorosa amarezza: "nemo propheta acceptus est in patria sua» (v. nemo propheta in patria), con riferimento, più che alla poco lusinghiera accoglienza fatta a lui dai Nazareni, all'atteggiamento generale degli Ebrei nei riguardi del suo messaggio...

automedicarsi v. rifl. [comp. di auto-1 e medicare] (coniug. come medicare). - Medicarsi da soli, senza l'intervento di un esperto....

medicatura s. f. [der. di medicare]. - Forma meno usata di medicazione, soprattutto nei sign. 1 e $3 . . .$.

immedicato agg. [comp. di in-2 e medicato, part. pass. di medicare], poet. - Propr., non medicato; fig., che non ha rimedio o sollievo: i. affanni (Leopardi)...

rimèdio (ant. remèdio) s. m. [dal lat. remedium, der. del tema di mederi «medicare, curare»]. - 1. Nome generico di ogni preparato medicinale, o cura medica, o altro mezzo, adoperati per combattere o alleviare le malattie: il chinino è un $r$. contro la malaria; per il raffreddore non c'è nessun r. specifico; il r. che ti ci vuole è un po' di riposo; tentare tutti i $r$. possibili. Con riguardo all'utilità, all'uso, agli effetti: r. efficace, opportuno, pronto, sicuro, risolutivo; $r$. generale, se... immedicàbile agg. [dal lat. immedicabillis], letter. - Che non si può medicare, quindi incurabile, insanabile: piaghe i.; male, ferita i. (anche fig., in senso morale); o che non ha sollievo: dolori i.; Tu piaga immedicabile Gemi ne' miei pensieri (Tommaseo).... 
medicheria s. f. [der. di medicare]. - Locale di un reparto ospedaliero, o clinico, o di una collettività (ospizî, carceri, ecc.), in cui si eseguono le medicazioni e si custodisce il relativo materiale necessario.... (http://www.treccani.it/vocabolari)

Come vediamo da questa struttura di famiglia di parole derivata costatiamo, che la parola di generazione iniziale che è medicare, che deriva dal latino, vediamo una situazione molto variegata di etimologia delle sue parole derivate; la maggior parte delle parole di questa famiglia deriva dal latino, oltre a medicare c'è anche medicazione, medicamento, medicatore, medicabile, medico, medica, medice, come si vede anche dalla struttura delle parole spiegate sopra. Invece la lista delle seguenti parole sempre derivate dal verbo medicare, che sono immedicato, automedicarsi, medicatura, medicheria, medico-sentinella e medico-clown sono parole, che hanno arricchito ulteriormente la famiglia della parola medicare non direttamente dal ceppo lessicale latino, ma in periodi relativamente recenti del medievo.

L'ottica dell'analisi, come vediamo, è basata anche sulla base della morfologia come inizio e dopo è supportata dalla base dei concetti della lessicologia e semantica. In effetti la formazione delle parole e la derivazione, caso specifico, come fenomeno linguistico, sia morfologico, lessicale e semantico, sembra essere piuttosto relativamente facile, analizzato e chiarito nella maggior parte della sua problematica. "La semantica è generalmente definita come lo studio del significato" (Lyons, J. (1980). Manuale di semantica. Bari:Laterza \& Figli. pagina 1)

La semantica in confronto alla morfologia e alla lessicologia è effettivamente più difficile e astratta da definire e discutere. In ultima analisi l'aspetto semantico della derivazione risulta essere il più interessante, il più utile anche il più difficile a farsi, per cui questo sarà uno dei binari principali, su cui ho in parte privilegiato questa analisi. Ecco cosa dice in merito, riportando quello, che dice un altro linguista;" Lo studio del significato è la parte più difficile della linguistica", scriveva Byussens nel 1960" ( Mounin, G. (1983). Guida alla semantica. Milano: Feltrinelli Economica, paggine 13).

Noi sappiamo, che la lessicalizzazione delle parole non è uniforme, essa viene vista in due categorie, sappiamo, che ci sono due tipi di studi lessicologici e linguistici in generale, sincronici e diacronici, che vuol dire, che sono sincronici quando appartengono a studi lessicali, che si verificano nel momento attuale della lingua, sono attivi al seno della lingua e originali nell'etimologia della lingua, che trattiamo, caso nostro l'italiano, e diacronici per studi linguistici appartenenti al passato.

La lingua italiana e il suo lessico può essere dunque, analizzato da due punti di vista: da

1. Lo studio sincronico (che deriva da syn-con, chronos-tempo, lo studio contemporaneo) la lingua viene studiata principalmente in un periodo relativamente recente, moderno, o meglio dire contemporaneo, come si presenta una lingua allo stato attuale. Questo tipo di studio suscita particolare interesse e viene preferito da molti linguisti.

2. Lo studio diacronico: ( che deriva da dia-attraverso, chronos, lo studio della lingua attraverso il tempo) si occupa dello studio del lessico visto nella sua evoluzione nel tempo, i cambiamenti morfo-lessicali e semantici, come sono avvenuti nel tempo, cioè un tuffo nel passato per vedere i cambiamenti morfo-lessicali e semantici nel processo evolutivo, per dedurre poi risultati e conclusioni importanti. Questo tipo di studio si considera più difficile del precedente e richiede maggior competenza linguistica e lessicale.

Questi due tipi di studio sono due metodi piuttosto complementari. II lessico della lingua italiana può essere studiato sia sul piano diacronico che, su quello sincronico, anzi bisogna dire, che gli studi lessico-semantici a volte è giusto che siano combinati, sia diacronicamente che sincronicamente, perché così magari si riesce a capire meglio una data problematica linguistica.

La nostra analisi non può essere esclusa dall'ottica diacronica, ma non perché figuri solo come referente scientifico, ma perché a volte risulterà effettivamente, inevitabile questo riferimento per sbloccare, analizzare, studiare e capire meglio una determinata problematica linguistica. Certamente la diacronia non ci condurrà a datare derivazioni, ma solo a decifrare, a interpretare linguisticamente un fenomeno e a capire meglio anche la sua semanticità. In merito a questo argomento possiamo citare l'illustrazione della parola medicare.

In italiano la derivazione è molto vasta come elementi formativi e produtività di parole, però il fenomeno della formazione delle parole racchiude i prefissi, i suffissi, gli infissi senza escludere però anche i casi con prefissi e suffissi zero, cioè senza elemnti formativi, ma di questo caso non posso occuparmene, solo per motivi di spazio e tempo.

I suffissi sono affissi, cioè elementi, che nel formare una parola si aggiungono alla parte finale di una radice 0 di un lessema, e questa unione non è casuale, ma ha le sue regole e tendenze a verificarsi. Questa unione come vedremo morfologicamente e formalmente, sembra una unione meccanica, ma in effetti questo succede soprattutto per ragioni semantiche, perché le parole hanno la motivazione d'uso per nascere, per il significato, è la principale ragione della loro nascita ed esistenza nel lessico di una lingua. 
I suffissi sono il tipo di affisso più usato nelle lingue. Lo stesso vale anche per l'albanese, cioè ci sono più parole derivate con suffissi, che con prefissi, perché ci sono più suffissi che prefissi in albanese e lo stesso dicasi dell'italiano, e sembra che, questo dovrebbe essere una tendenza normale per molte lingue. Effettivamente è realmente maggiore la produzione delle parole derivate con suffissi che prefissi, sia in italiano che in albanese. "I sostantivi nell'albanese di oggi sono una delle parti del discorso, che si aggiungono e si arricchiscono continuamente con nuove parole" ( La grammatica della Lingua Albanese, pagina 132)

In italiano bisogna affermare, che quanto alla derivazione il caso della produzione derivazionale così particolare $\mathrm{e}$ produttivo, perché è molto vasto e complesso sia per quanto riguarda il numero degli elementi formativi, che per la loro diversità semantica e produttività. In questo capitolone della formazione delle parole e della derivazione delle parole con affissi, gli alterati si inseriscono in questo contesto linguistico, però citati solo per argomento e appartenenza qui, perché gli alterati, presenterebbero una grande problematicità e sottosistema lessicale tale che, meriterebbe una trattazione a parte e lunga e non è il caso di fermarsi su questo più a lungo. Essi si suddividono in quattro piccoli sottogruppi, alterati diminutivi, accrescitivi, vezzeggiativi e peggiorativi. Gli alterati si formano assommandosi a fine parole esistenti, che può essere sostantivo, aggettivo avverbio o verbo con lo scopo di solo modificare il significato e di non cambiarlo, trasformarlo, ma di darle una sfumatura specifica. II caso di alterati di sostantivi e il più produttivo degli altri e per giunta il più intricato per la sua semantica. Suppongo, che in italiano il caso della derivazione, che riguarda gli alterati è abbastanza peculiare, perché è un sottosistema perfettamente funzionante, vasto ricco e sempre potenziale nella sua produzione. Dopo questa doverosa interpretazione sugli alterati torniamo al nostro caso.

II numero dei suffissi è numericamente superiore a quello dei prefissi: i prefissi dell'italiano sono circa ottanta, i suffissi derivazionali (compresi gli alterativi) sono più del doppio. Questa situazione è relativamente uguale alle altre lingue lingue neolatine.

La maggior parte dei suffissi derivazionali italiani provengono dal latino (per es., -aio lat. -ariu(m): libraio; -evole lat. -ibile(m): pieghevole, identificabile; -mento lat. -mentu(m): cambiamento).

Ci sono suffissi di origine latina risalenti al greco (per es., -ico lat. -icum gr. -ikós: storico; -ismo lat. -ismu(m) gr. ismós: marxismo, capitalismo, idealismo, materialismo; -ista lat. -ista(m) gr. -istếs: pianista, musicista, chitarrista, violinista, leninista; -izzare lat. tardo -izare gr. -ízō: socializzare, formalizzare, razionalizzare, nazionalizzare ecc ).

I suffissi di formazione italiana sono in maggioranza originati da materiali di origine latina (per es., -ata, manata, panciata; -ato, fortunato); è di formazione italiana un certo numero di suffissi alterativi (per es., -occio, figlioccio, belloccio, -accione, spendaccione).

La maggior parte dei suffissi di origine straniera proviene dal francese -age (per es., -aggio montaggio, monitoraggio, brigantaggio ecc., caso lo vuole che il suffisso -aggio italiano coincide con quello francese e si adotta facilmente lessicalmente ed è molto produttivo come suffisso anche in italiano per cui l'addattamento delle voci francesi derivate col suffisso -age vengono naturalmente accolte, addattate e adottate. "Siamo convinti, insomma, che in una situazione di sempre più incalzante concorrenza "esterna" all'italiano, ci sia almeno bisogno di alcuni segnali di disgelo nel sistema ereditato dal nostro passato; un passato che per secoli non ha potuto giovarsi di un forte uso parlato, naturale fattore di assestamento delle lingue. ( Dizionario della Lingua Italiana, Sabatini, Coletti, pagina III).

I prefissi e i suffissi vengono a derivare nuove parole modificando, arricchendo e precisando il significato originale creando nuove parole nella lingua e sono un importante fattore di ricchezza della lingua, come lo abbiamo anche già precedentemente affermato. II greco meno e il latino di più forniscono a livello odierno all'italiano e di solito, a molte lingue parole a suo tempo effettivamente tali semanticamente parlando nelle rispettive lingue, ma a distanza di tempo queste parole hanno perso la loro naturale esistenza nelle loro lingue e sono sopravissute oggi come elementi formativi, cioè come prefissi e suffissi necessari alla formazione di parole moderne. II ricorso a questi espedienti lessicali in molte lingue risulta essere indispensabile e pare, come fosse solo monopolio del fornimento di queste due lingue classiche. Queste ex parole oggi funzionanti come elementi formativi sono a tutti gli effetti parte del grande sistema italiano della formazione delle parole con prefissi e suffissi. Ecco qui sotto vediamo una lista esemplare di queste parole greche e latine usate come prefissi.

Parole latine usate come prefissi:

Arbor-(i) albero, arboricultura, deci-(dieci) decimetro, multi (numeroso) multiforme, multinazionale, onni- (tutto) onnipotente, radio- (raggio) radiologia, quinque- (cinque) quinquennale.

Parole greche usate come prefissi:

aero- (aria) aerologia, aeronautica; antropo- (umano) antropofago; archeo-, antico, archeologia, auto- (da solo) automobile; baro- (pesante) baromente; bibblio-(libro) bibblioteca, bibbliografia; bio-(vita) biologia; caco-(cattivo,brutto) cacofonia; cinamat-(a)-(movimento)-cinematografo; clepto-(furto) cleptomania; cosmo-(mondo) cosmonauta; cripto-critto 
(nascosto) crittogramma, crono-(tempo) cronometro; dattilo-(dito) dattilografo; demo- (popolo) democrazia; dinam(o)(forza) dinamometro; fil(o)- filiforme, filodifusione, filosofia, filantropo; fon(o)- (voce) fonografo, fonetico; foto-(luce) fotografia; eco- (casa ambiente) economia, ecologia; elio- (sole) eliocentrico; emo- emat(o)- (sangue) emofilia, ematologia; gaster-, gastro- gasteropodo, gastronomia; geo- (terra) geologia; idr(o)- idroscalo, idrico; ippo- (cavallo) ippodromo; iso-(uguale) isoterme, isoscele; lito-(pietra) litografia; macro-(grande) macrecefalo; mega- (grande) megalomane; necro-(morte) necroforo; neo-(nuovo) neologismo; nevr- (neuro,nevro) nevralgia, neuratico, omo- (simile) omogeneo; oro- (montagna) orografia; orto- (frutta) ortografia, ortopedia; paleo- (antico) paleolitico; pato-(sofferenza) patologia; ped- (bimbo) pediatria, penta- (5) pentametro; piro-(fuoco) pirotecnico; pneuma- (aria) pneumatica; poli(numeroso) poliglotta; pseudo- (falso) pseudonimo; tecn(o)- (arte) tecnico; tele- (lontano o distanza) televizione; termo(calore) termometro; tipo- (carattere) tipografo; topo- (luogo) topografia; zoo- (animale) zoologia.

Ecco come definisce esattamente Giacomo Devoto il significato del suffisso:

SUFFISSO. - È un morfema (v. morfologia: Linguistica) che segue al semantema direttamente aggiunto, separato da altri morfemi o da semimorfemi. Si distingue così da prefisso (messo davanti) e infisso (messo in mezzo), s'intende nel semantema. I suffissi possono essere, a rigore tanto di derivazione quanto di flessione: in quest'ultimo caso si usa il termine di desinenze. I suffissi sono primari o secondari a seconda, siano in contatto diretto o meno diretto col semantema; per es.: particolarità, part- semantema originario, icol- suffisso primario ereditato dal latino, -ar- suffisso primario che forma aggettivi, -ità suffisso secondario che forma astratti.

L'etimologia della stessa parola, suffisso suggerisce, che è s. m., derivato dal lat. suffixus «attaccato sotto», part. pass. di suffigĕre: verbo suffiggere.

La formazione delle parole e concretamente la derivazione con suffissi e prefissi è un segno della legge dell'economia della lingua, perché, se non ci fosse la formazione delle parole con la derivazione allora per ogni concetto nuovo ci dovrebbe essere una parola nuova e questo significherebbe una nuova combinazione fonematica e continuando così, il numero delle parole per ogni lingua sarebbe per più grande. " La vitalità della formazione delle parole si manifesta anche con lo sviluppo, nel linguaggio dei media..."( Alberto A. Sobrero. Introduzione all'italiano contemporaneo, pagina 344)

Collegando all'idea del risparmio della lingua, non dobbiamo dimenticare, che la polisemia è una delle vie più originali linguistiche, che risparmia la creazione di nuove parole, così attraverso essa invece, che si creino nuove parole fonematicamente, cosa assolutamente non sempre possibile e comodamente facile a realizzarsi, risolve i problemi semantici aggiungendo a parole, già esistenti nuovi significati, sovracariccando semanticamente, parole con significati in uso nella lingua. La lingua a quanto pare per i concetti nuovi avrebbe difficoltà a creare sempre nuove parole per ogni concetto nuovo, ma molte volte risolve molto meglio con questi due sistemi e meccanismi; con la formazione delle nuove parole, con la suffissazione e la prefissazione e la polisemia. E questo modo semplificherebbe e faciliterebbe, come pare anche la gestione semantica delle parole. "Così la semantica linguistica si definisce come lo studio del contenuto di segni e combinazioni di segni, che sono possibili o addirittura appaiono in lingue naturali" ( Introduction à la lexicologie du français, Nonda Varfi ). Perché, come sappiamo, pure, per ogni significato non ci sono parole nuove, se no allora avremmo un numero $4-5$ forse più volte maggiore delle parole esistenti in ogni lingua.

Essendo l'italiano una lingua neolatina, e siccome già in latino avevamo un sistema intero e completo di meccanismi morfo-lessicali, che funzionavano così bene in numero e in maniera perfetta. Allora tutto questo meccanismo linguistico si può immaginare, che sia facilmente passato in italiano. Gran parte dei suffissi e prefissi latini sono presenti e produttivi oggi in italiano. Ovviamente noi sappiamo, che questi mezzi formativi non sono elementi puramente semantici, questi non hanno significato, semanticità, però non si può dire, che essi siano completamente privi di semanticità, senza una funzione, funzionalità semantica. Questo è vero e verificabile perché, se vediamo lo stesso suffisso 0 prefisso attaccato a un morfema costatiamo, che una certa tendenza semantica simile, se non uguale, si verifica più o meno come una tendenza semantica puntuale. Questa tendenza semantica non sempre identica però, è abbastanza simile. Così solo a mo' d'esempio, possiamo citare il suffisso nominale -ismo, che spesso troviamo in parole tipo perbenismo, comportamentismo, comunismo, capitalismo, consumismo, realismo, esistenzialismo, assenteismo, edonismo, illuminismo, occultismo, oscurantismo ecc. E vediamo un po' l'aspetto semantico, come si comporta il suffisso attaccato al tema, con cui forma la parola. Vediamo la semantica complessa delle parole derivate col suffisso -ismo, solo per stimolare la curiosità sulla particolarità della definizione lessicografica, che ne presentano queste parole.

La parola perbenismo con connotazione polemica, modo di comportarsi di chi, vuole apparire persona perbene, seguendo con qualche ostentazione le norme della morale comune 0 uniformandosi a quelle della classe sociale dominante: condotta ispirata a un ipocrita $p$.; tutto era lì per dimostrare il p. immacolato della famiglia Cuccoli (Palazzeschi); urlavo ... che non ne potevo più del p., che la morale borghese non faceva per me (Moravia)... 
Vediamo adesso la parola comportamentismo. In psicologia, indirizzo di studio (detto anche, con adattamento del corrispondente termine ingl., behaviorismo), che estende i metodi osservativi della psicologia animale e l'analisi delle correlazioni individuo-ambiente a tutta la psicologia, compreso l'uomo, contestando l'uso dell'introspezione e contrapponendosi a impostazioni che si fondano su entità come stati mentali e coscienza (v. mentalismo), pulsioni, istinti, ecc.

Nel caso di perbenismo riconosciamo l'elemento semantico di " modo di comportarsi di chi vuole apparire persona perbene, seguendo con qualche ostentazione le norme della morale comune 0 uniformandosi a quelle della classe sociale dominante", che è presente anche nel caso di comportamentismo che troviamo "correlazioni individuo-ambiente a tutta la psicologia, compreso l'uomo ".

Per non parlare poi di parole, che perfettamente nella stessa gamma semantica addirittura tipo capitalismo, feudalesimo, socialismo, comunismo, dove tutte e quattro hanno quasi la stessa spiegazione iniziale tipo sistema politico, economico, sociale e culturale poi seguono i dettagli specifici per ogni parola, così per capitalismo abbbiamo anche: "Concezione capitalistica dell'economia che si propone di contemperare esigenze competitive e coesione sociale".

Socialismo;"concezione della società che propugna la soppressione di ogni privilegio e mira a instaurare la piena uguaglianza dei suoi membri". In questa accezione generale il termine si riferisce anche a quei movimenti e a quelle teorie sorti prima della rivoluzione industriale, che puntavano all'eguaglianza totale nella comunione dei beni.

Tra questi elementi formativi vale a dire, che ci saranno alcuni suffissi, che avranno un valore terminologico, si useranno cioè solo in determinati contesti specifici, scientifici e ne possiamo citare anche solo come esempio anche il suffiso suddetto -ismo. " ...è inutile e indesiderabile assumere, che le parole abbiano un significato pienamente determinato." ( Lyons John Introduzione alla linguistica teorica, III la semantica, pagina 544)

I suffissi italiani nominali in funzione all'origine possono essere, se derivano da verbi si dicono deverbali, denonimali se deriva da nomi, deaggettivali, se derivano da aggettivi. la base da cui derivano ovviamente ha una certa importanza, perché semantimene la parola derivata mantiene i legami con l'origine. Vediamo a mo' d'esempio, dei casi del tipo di derivazione deverbale.

Suffissi nominali deverbali;

In italiano sono: -aggio (suffisso d'origine francese; se parole medievali come coraggio e retaggio non sono ormai analizzabili, forse per coraggio potremmo supporre, che deriva anche alla lontana da cuore e direttamente da core, che è una forma letteraria accorciata di cuore e piu' il suffisso -aggio e deriva coraggio, ma in genere questi casi di derivazione e lessicalizzazione sono piu' fossilizzati e per questo diacronici e meno interessanti. Diverso è il caso delle formazioni moderne e diciamo " fresche "come abbordare abbordaggio, tatuare tatuaggio); -ando, -anda (come nel gerundio latino esprime l'idea del dovere: esecrare esecrando, 0 anche solo dell'imminenza: laureare laureando); -ante, -ente (corrispondenti al participio presente latino, danno luogo a un sostantivo riferito a una persona che compie una certa azione: parlare parlante), ma anche a un prodotto, che ha un dato uso (sbiancare sbiancante) 0 a una nozione ché astrattacamente (muovere, movente), -ato, -ata, -ito, -ita, ecc. (suffissi propri del participio passato adottati in forme sostantivali: lavare lavata, tenere tenuta). II caso dei participi presenti e quello dei participi passati, usati lessicalmente, come sostantivi o aggettivi è un caso molto interessante e meriterebbe una lunghissima trattazione sua, tale è la sua complessità e consistenza, ma basta solo menzionarla come importanza. -anza, -enza (molto frequenti nella poesia antica per influsso del modello d'oltralpe, danno luogo a sostantivi astratti: mancare mancanza); -io (luccicare luccichio, pigolare pigolio); -ino, -ina (spazzare spazzino); -mento (il suffisso fondamentale, insieme a -zione e a -sione: affondare affondamento, abbellire abbellimento); -one, -ona (mangiare mangione); -toio, torio, -toia, -toria (mangiare mangiatoia, parlare parlatorio); -ura (aggiunto alla base del participio passato: bruciare (bruciato) bruciatura); -zione, -sione (demolire demolizione, riprendere riprensione); inoltre -tore, -trice (con valore di agente: truccare truccatore, bandire banditore). Ci sono poi alcuni casi di sostantivi deverbali senza suffisso o, come si dice, a suffisso zero: ad esempio deliberare delibera (per deliberazione) o il recente utilizzare utilizzo (per utilizzazione), diciamo zero, perché in effetti quando diciamo il verbo lui delibera, che è terza persona, e io utilizzo, che è la prima persona qui di affissi, non si può parlare perché, poi i sostantivi siddetti coincidono perfettamente con queste parole, che sono delibera e utilizzo. Di esempi così, in italiano, se ne trovano a iosa, ma basta anche solo questi casi per illustrare la situazione.

\section{Conclusioni}

Questo argomento da me trattato è un un argomento molto trattato e analizzato dalla linguistica italiana, ma la mia visione ha avuto la pretesa modesta di essere piuttosto originale nel trattarlo, premetto subito che, non avevo assolutamente l'intenzione di gettare luce sul completo e totale questo enorme capitolo della lingua italiana sulla 
formazione delle parole, ma ho pensato di apportare un mio modesto contributo originale, nel vedere questa problematicità linguistica, che resta sempre interessante e utile da conoscere. Non poteva essere assolutamente esaustiva, come trattazione se no, ci vorrebbero centinaia di pagine per considerare sotto tutti gli aspetti questa problematicità, magari sempre sulla mia ottica lessico-linguistica. Lo scopo era di accendere dei proiettori potenti, su problemi linguistici e semantici quali la derivazione delle parole in italiano e meno sulla composizione. Indirettamente si è mirati a facilitare, chi vorebbe a interessarsi di problemi simili, a sbriciolare problemi complicati lessicali e semantici a risolverli in maniera capace. La trattazione su alcuni argomenti e dettagli si è fermata di più, su altri meno e qualcuno affatto; questo, non per opportunismo di analisi, ma partendo da alcuni casi specifici si è inteso arrivare a conclusioni più generalizzanti, questo anche per via della "cornice" della collana, riferendomi allo spazio ovviamente. Questa fa parte certamente anche di un mia personale preferenza di analisi, perché di solito ci sono studi, che si occupano di minuzie e dettagli tutto il loro studio, con tutto il rispetto di questo, come possono essere anche studi preferenziali, sulla generalizzazione di una fenomenologia di qualsiasi scienza, che tratta argomenti suoi. Infine posssiamo pure suggerire, che questa analisi aiuterebbe probabilmente, sopratutto chi, insegnerebbe l'italiano e non solo italiano in generale e anche chi apprenderebbe l'italiano 0 anche altre lingue europee in particolare.

\section{References}

Lo Zingarelli 1995. Vocabolario della lingua italiana. Zanichelli Bologna, http://www.treccani.it

Dizionario della Lingua Italiana, Sabatini, Coletti. Rizzoli Larousse. Milano 2003

La grammatica della Lingua albanese, Accademia delle Scienze della Repubblica D'Albania, Istituto della Linguistica e della Lettertura, Tirana 1995

Mounin, G. (1983). Guida alla semantica. Milano: Feltrinelli Economica.

De Voto-Oli (2004). Dizionario della Lingua Italiana. Firenze: Felice Le Monnier, Edmund Le Monnier S.p.A.

Nonda VARFI, Introduction à la lexicologie du français, Lausanne, Ecole de français moderne de l'Université de lausanne, 1999, 29 pp.

Lyons, J. (1980). Manuale di semantica. Bari:Laterza \& Figli.

Lyons John Introduzione alla linguistica teorica, III La semantica. Bari:Laterza \& Figli 1981

Alberto A. Sobrero. Introduzione all'italiano contemporaneo. vol. I. Le strutture. Editori Laterza 1993.

Chierchia, G. (1997). La semantica, le strutture della lingua. Bologna: Mulino.

Serianni Luca, Grammatica italiana. Editore: UTET Università, De Agostini, Scuola, Novara. 2006) 
